underrecognized form of substance abuse with serious acute and chronic toxicities. Acute symptoms of toluene toxicity include dizziness, euphoria, headache, vomiting, vertigo, convulsions, and loss of consciousness, sometimes preceded by delirium. Chronic toluene abuse causes headache, muscle weakness, peripheral neuropathy, nervousness, anemia, petechiae, abnormal bleeding, bone marrow aplasia, irreversible encephalopathy, and renal tubular acidosis. Following an initial report of a "Fetal solvents syndrome"(Toutant C, Lippman S. Lancet 1979;1:1356), the effects of toluene on the fetus have been described infrequently. This Arizona University study of a fetal toluene syndrome compares findings in their 18 patients with others in the literature and with the fetal alcohol syndrome. The authors conclude that the craniofacial teratogenetic effects of toluene and alcohol have a common mechanism.

An additional 6 cases of toluene embryopathy are reported from the Denver General Hospital, Colorado (Arnold GL et al. Pediatrics Feb 1994;93:216). Only one was exposed to alcohol as well as toluene.

\title{
LEAD POISONING RISK ASSESSMENT: BLOOD LEAD SCREENING
}

The Centers for Disease Control and Prevention (CDC) lowered the blood lead level $(\mathrm{BPb})$ considered a toxic risk to children from $25 \mathrm{mcg} / \mathrm{dL}$ to 10 $\mathrm{mcg} / \mathrm{dL}$ in Oct 1991. A five-item questionnaire is now completed at regular office visits for all children from 6 months to 6 years of age to identify those at high risk of lead exposure. In some States, including Illinois, all children are required to have $\mathrm{BPb}$ testing at 12 months of age or before entering a statelicensed day care, preschool, or kindergarten. High risk infants must be tested at 6 months and biannually.

A study to determine the efficacy of the questionnaire and prevalence of elevated $\mathrm{BPb}$ in 1393 suburban children at 12 and 24 months of age is reported from the Department of Pediatrics, Children's Memorial Hospital, Northwestern University, Chicago, Illinois. A venous $\mathrm{BPb}=/>10 \mathrm{mcg} / \mathrm{dL}$ was found in $2.1 \%$, and none was $>30 \mathrm{mcg} / \mathrm{dL}$. CDC and Illinois screening tests failed to predict high risk exposure in 9 of $29(31 \%)$ children with elevated $\mathrm{BPb}$. Living in a pre-1960 house, a question not included in CDC or Illinois screening, was most predictive, with positive correlation in 24 of $29(83 \%)$ children with elevated $\mathrm{BPb}$ levels. The authors point out that optimal risk assessment questions may vary in different areas and populations. (Binns HJ et al. Is there lead in the suburbs? Risk assessment in Chicago suburban pediatric practices. Pediatrics Feb 1994;93164-171). (Reprints: Helen J Binns MD, Children's Memorial Hospital, 2300 Children's Plaza, Chicago, IL 60614).

COMMENT. Questions about the home environment were the most sensitive indicators of elevated lead levels in a similar study reported from the California Pacific Medical Center, San Francisco, CA. (Tejeda DM et al. Do questions about lead exposure predict elevated lead levels? Pediatrics Feb 1994;93:192-194). An abbreviated screening using only the first three items was as effective as the complete CDC questionnaire in a study at the University of Rochester, NY.(Schaffer SJ et al. Lead poisoning risk determination in an urban population through the use of a standardized questionnaire. Pediatrics Feb 1994;93:159-163). Selective screening with a community-specific questionnaire is proposed following a study at the Gunderson Clinic, La Crosse, WI, which found a great variability in prevalence of elevated $\mathrm{BPb}$ between clinics 
even within a homogeneous community. (Rooney BL et al. Development of a screening tool for prediction of children at risk for lead exposure

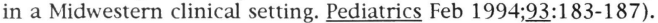

A survey of 556 pediatricians in Virginia revealed an overall deficiency in physicians' knowledge of lead poisoning with specific deficiencies in knowledge of the literature. Subspecialists scored lower than primary care pediatricians. (Bar-on ME, Boyle RM. Are pediatricians ready for the new guidelines on lead poisoning? Pediatrics Feb 1994; $93: 178-182$ ). Since $17 \%$ of all children $<7$ years in the USA are reported to have elevated $\mathrm{BPb}$ levels known to increase the risk of cognitive and behavioral deficits, both physician and parent awareness of the environmental sources, symptoms, and long-term hazards of lead is an urgent priority. (Millichap JG. Environmental Poisons in Our Food. Chicago, PNB Publishers, 1993).

\section{MUSCLE DISORDERS}

\section{CONGENITAL NEMALINE MYOPATHY}

A female neonate with a rapidly fatal course of nemaline myopathy is reported from the University of Siena, Italy. Positive pressure ventilation was required and postasphyxia suspected. Despite improved cardiorespiratory function, severe hypotonia, muscle weakness and areflexia persisted. At 2 months, fractures of both femurs and left humerus were noted, and a myopathy was considered in diagnosis. Muscle biopsy of quadriceps showed rod-shaped nemaline bodies. The infant died at 4 months of pneumonia. Nemaline bodies were found in diaphragm, intercostal, psoas, and quadriceps muscles. The heart was also involved. The parents were healthy and their muscle biopsies normal. (Buonocore $G$ et al. A new case of severe congenital nemaline myopathy. Acta Paediatr Dec 1993; $122: 1082-4)$. (Respond: Dr G Buonocore, Division of Neonatology, University of Siena, via P Mascagni, 4653100 Siena, Italy).

COMMENT. Persistence of severe hypotonia in a neonate, together with dependence on assisted ventilation, should prompt investigation of a possible myopathy.

Intranuclear rods were present in muscle fibers of one infant with a rapid, fatal course of nemaline myopathy but were absent in the muscles of seven patients with a benign course, in a study reported from the Departments of Neurology and Pathology, University of Rochester Medical Center, NY. (Rifai Z et al. Intranuclear rods in severe congenital nemaline myopathy. Neurology Nov 1993;43:2372-2377). The presence of intranuclear rods represents a marker for the severe form of congenital nemaline myopathy.

The clinical manifestations of three forms of nemaline myopathy are reported as follows: 1) severe neonatal form, with hypotonia, feeding and respiratory difficulties, and death in infancy; 2) nonprogressive or slowly progressive form, presenting in infancy or early childhood with delayed motor milestones and facioscapuloperoneal weakness; and 3) adult-onset form, with a progressive proximal weakness. The term "congenital nemaline myopathy" is applied to forms 1) and 2). The authors caution that the neonatal type is not invariably fatal, and improvement may occur, or alternatively, deterioration may follow an initial stable course. 